\title{
The cobordism of Real manifolds
}

\author{
by
}

Po H u (Chicago, IL)

\begin{abstract}
We calculate completely the Real cobordism groups, introduced by Landweber and Fujii, in terms of homotopy groups of known spectra.
\end{abstract}

1. Introduction. The notion of Reality was first introduced by Atiyah [4] for $K$-theory. A Real bundle over a $\mathbb{Z} / 2$-space is a complex bundle, together with a complex antilinear $\mathbb{Z} / 2$-action compatible with the $\mathbb{Z} / 2$-action on the base. A Real manifold is a $\mathbb{Z} / 2$-equivariant smooth manifold with a Real structure on its normal bundle. The cobordism of Real manifolds was first considered by Landweber [14] and later Fujii [12]. Landweber [15], Araki [3], and Fujii [10] also introduced the Real Thom spectrum $M \mathbb{R}$, a $\mathbb{Z} / 2$-equivariant spectrum indexed over the complete universe in the sense of Lewis, May and Steinberger [16], whose homotopy groups were calculated by Araki (see [13]). Landweber [14, 15] and Fujii [10] obtained partial results relating the Real cobordism group, which we denote by $\Omega_{\star}^{\text {Real }}$, to $\pi_{\star} M \mathbb{R}$. Interest in Real cobordism increased recently in connection with a Real version of the Adams-Novikov spectral sequence (see [13]).

In this note, we completely calculate $\Omega_{\star}^{\text {Real }}$ in terms of $M \mathbb{R}$ and other known spectra. It should be pointed out that our treatment of geometric Real cobordism here is a fairly straightforward application of the methods of Conner and Floyd [7], Costenoble and Waner [8], tom Dieck [9], and Wasserman [20]. Nevertheless, the problem clearly was considered, though not completely solved, in the literature ([14], [12], [15], [3], [10]). The purpose of this paper is to record the answer, with a complete, self-contained proof.

Ideally, we would like to show that the Real cobordism groups of compact Real manifolds are $M \mathbb{R}_{\star}$, in analogy with the case of complex cobordism. However, as usual this turns out to be false, due to the lack of transversality.

1991 Mathematics Subject Classification: Primary 55N22; Secondary 55P91.

The author is partially supported by a NSF Postdoctoral Research Fellowship. 
Hence, we consider first the case of free Real cobordism of free Real manifolds (see also Fujii [12] and Landweber [15]). There is the following result, analogous to the case of equivariant cobordism:

Proposition 1. The free Real cobordism groups of free compact Real manifolds are $\left(M \mathbb{R} \wedge E \mathbb{Z} / 2_{+}\right)_{\star}$.

For Real cobordism of general Real manifolds, consider the cofibration sequence

$$
E \mathbb{Z} / 2_{+} \rightarrow S^{0} \rightarrow \widetilde{E \mathbb{Z} / 2}
$$

After smashing it with $\Sigma^{-l \alpha} M \mathbb{R}$ and taking $\mathbb{Z} / 2$-fixed points, we obtain the cofibration sequence of nonequivariant spectra

$$
\left(\Sigma^{-l \alpha}(M \mathbb{R} \wedge E \mathbb{Z} / 2)\right)^{\mathbb{Z} / 2} \rightarrow\left(\Sigma^{-l \alpha} M \mathbb{R}\right)^{\mathbb{Z} / 2} \rightarrow \Phi^{\mathbb{Z} / 2}\left(\Sigma^{-l \alpha} M \mathbb{R}\right)
$$

where $\Phi^{\mathbb{Z} / 2}$ denotes the geometric fixed points in the sense of [16]. These can be found by taking fixed points on the prespectrum level. For $M \mathbb{R}$, the spaces are of the form $T\left(\left.\gamma_{\text {Real }}^{n}\right|_{B \mathbb{U}(n)}\right)$. Taking fixed points gives $T\left(\left.\gamma^{n}\right|_{B O(n)}\right)$, and suspension by $l \alpha$ does not affect the geometric fixed points, so

$$
\Phi^{\mathbb{Z} / 2} \Sigma^{-l \alpha} M \mathbb{R}=\Phi^{\mathbb{Z} / 2} M \mathbb{R}=M O .
$$

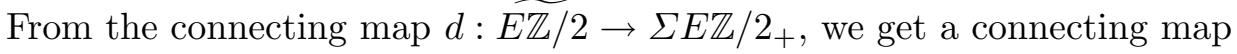

$$
d: M O \rightarrow \Sigma\left(\Sigma^{-l \alpha}(M \mathbb{R} \wedge E \mathbb{Z} / 2)^{\mathbb{Z} / 2}\right) .
$$

Now let $M$ be a Real manifold of dimension $k+l \alpha$, with fixed point set $M^{\mathbb{Z} / 2} \cdot M^{\mathbb{Z} / 2}$ is a real submanifold with dimension $k$, and so specifies an element of $\pi_{k}\left(\Sigma^{-l \alpha} \Phi^{\mathbb{Z} / 2} M \mathbb{R}\right)=\pi_{k} M O$. However, this is not all of the structure present on $M^{\mathbb{Z} / 2}$ : there is also an $l$-dimensional bundle $\eta$ such that $\eta \oplus \nu_{M^{\mathbb{Z} / 2}}$ is trivial. We will call such manifolds $l$-trivial. While deferring the precise definition of cobordism of $l$-trivial manifolds to the next section, we have the following result.

Proposition 2. The cobordism groups of compact l-trivial real manifolds are the homotopy groups of $\Sigma^{-l} T\left(\left.\gamma^{l}\right|_{B O(l)}\right)$.

There is a stabilization map $\iota: \Sigma^{-l} T\left(\left.\gamma^{l}\right|_{B O(l)}\right) \rightarrow M O$. Thus, composing with the connecting map $d$, we get a map

$$
\delta: \Sigma^{-l} T\left(\left.\gamma^{l}\right|_{B O(l)}\right) \stackrel{\iota}{\rightarrow} M O \stackrel{d}{\rightarrow} \Sigma\left(\Sigma^{-l \alpha}(M \mathbb{R} \wedge E \mathbb{Z} / 2)^{\mathbb{Z} / 2}\right) .
$$

Let $M \mathbb{R}_{l}$ be the homotopy fiber of the map $\delta$. Also, denote the Real cobordism group of Real manifolds of dimension $k+l \alpha$ by $\Omega^{\text {Real }}(k+l \alpha)$. The following theorem is the main result of this paper.

TheOREM 3. For a given $l>0, M \mathbb{R}_{l}$ is the cobordism spectrum of compact Real manifolds of dimension $k+l \alpha$. In other words,

$$
\Omega^{\text {Real }}(k+l \alpha) \cong \pi_{k} M \mathbb{R}_{l} .
$$


In Section 2, we will make precise the notions of Real cobordism of Real manifolds and free Real cobordism of free Real manifolds, as well as that of $l$-trivial manifolds and their cobordism. Section 3 will be devoted to the proof of Proposition 1; and the main result will be proven in Section 4.

2. The main definitions. Let $\alpha$ denote the unique nontrivial irreducible representation of $\mathbb{Z} / 2$. A Real manifold (with boundary) is a manifold (with boundary) $M$ with a smooth action of $\mathbb{Z} / 2$, together with a Real bundle $\nu$ of dimension $n(1+\alpha)$ over $M$, and an isomorphism

$$
\tau_{M} \oplus \nu \cong m+p \alpha
$$

where $m+p \alpha$ denotes the trivial $\mathbb{Z} / 2$-equivariant bundle isomorphic to direct product with the representation $\mathbb{R}^{m+p \alpha}$. The Real dimension of $M$ is then defined to be $m+p \alpha-n(1+\alpha)$. $M$ is a free Real manifold if the action of $\mathbb{Z} / 2$ on $M$ is free.

A compact Real manifold $M$ can be embedded smoothly and equivariantly into $\mathbb{R}^{m+p \alpha}$. Then $\nu$ is the normal bundle of $M$ in $\mathbb{R}^{m+p \alpha}$. Note that there may be Real bundles $\nu, \nu^{\prime}$ of dimensions $n(1+\alpha), n^{\prime}(1+\alpha)$ on $M$ such that

$$
\tau_{M} \oplus \nu=m+p \alpha, \quad \tau_{M} \oplus \nu^{\prime}=m^{\prime}+p^{\prime} \alpha .
$$

Hence, there may be more than one Real structure on $M$, with possibly different Real dimensions $k+l \alpha$ and $k^{\prime}+l^{\prime} \alpha$, where $k+l=k^{\prime}+l^{\prime}$ is the real dimension of $M$. An actual example of this ambiguity of Real dimension is the manifold $S(n \alpha)$, of nonequivariant dimension $n-1$. Note that $\tau_{S(n \alpha)} \oplus$ $1=n \alpha$, so $S(n \alpha)$ is a Real manifold of dimension $n \alpha-1$. On the other hand, by division algebra theory, for $n=2,4,8$, we have $\tau_{S(n)} \cong n-1$, and hence $\tau_{S(n \alpha)}=\tau_{S(n)} \alpha \cong(n-1) \alpha$ for $n=2,4,8$, giving $S(n \alpha)$ a Real structure of dimension $(n-1) \alpha$.

Let $M$ be a Real manifold with boundary of dimension $k+l \alpha$, and let $\mu$ be the trivial 1-dimensional collar bundle of $\delta M$ in $M$. If we choose $m, p$ sufficiently large, then on $\delta M$, the complement of $\mu$ in $\left.(m+p \alpha)\right|_{\delta M}$ is trivial of dimension $(m-1)+p \alpha$. So we have an isomorphism

$$
\left.\tau_{\delta M} \oplus \nu\right|_{\delta M} \cong(m-1)+p \alpha
$$

giving $\delta M$ an induced structure of a Real manifold of dimension $(k-1)+$ $l \alpha$. Again, choosing $m, p$ sufficiently large, we see that this induced Real structure is unique.

As in the nonequivariant case, given a Real manifold $M$ with its structure given by the Real normal bundle $\nu$ and isomorphism $\tau_{M} \oplus \nu \cong m+p \alpha$, we can define the Real manifold $-M$ as the same manifold and normal bundle, but reversing one chosen coordinate in the trivialization isomorphism. 
Definition 4. A compact Real manifold $M$ of dimension $k+l \alpha$ is Real cobordant to 0 if there is a Real manifold with boundary $P$ such that the boundary of $P$ is $\delta P=M$. If $M$ is free, then it is free Real cobordant to 0 if there is a free Real manifold $P$ with $\delta P=M$. Two compact Real manifolds $M, N$ of dimension $k+l \alpha$ are cobordant if $M \amalg(-N)$ is Real cobordant to 0 , and free Real cobordant if $M \amalg(-N)$ is free Real cobordant to 0 .

Real cobordism and free Real cobordism are equivalence relations. The cobordism classes of compact Real manifolds of dimension $k+l \alpha$ form an abelian group under disjoint union, which is the Real cobordism group of dimension $k+l \alpha$. Similarly, the free cobordism classes of free compact Real manifolds of dimension $k+l \alpha$ form the free Real cobordism group of dimension $k+l \alpha$.

We also make precise the definition of $l$-trivial manifolds.

DeFinition 5 . For a given $l>0$, an $l$-trivial bundle is a real bundle $\xi$ (i.e. an $\mathbb{R}^{n}$-bundle) such that $\xi \oplus \eta$ is trivial for some $l$-dimensional real bundle $\eta$. An l-trivial manifold (with boundary) is a real manifold (with boundary) $N$, with a normal bundle $\nu$ and an $l$-dimensional real bundle $\eta$ that gives an $l$-trivial structure to $\nu$, i.e. isomorphisms

$$
\tau_{N} \oplus \nu=m, \quad \eta \oplus \nu=p
$$

where $m, p$ denote trivial real bundles of these dimensions.

Note that if $M$ is an $l$-trivial manifold with boundary of dimension $k$, with $l$-bundle $\eta$, then $\delta M$ is an $l$-trivial manifold of dimension $k-1$, with l-bundle $\left.\eta\right|_{\delta M}$.

Given an $l$-trivial manifold $M$ with normal bundle $\nu$ and $l$-bundle $\eta$, we define $-M$ as the same manifold and bundles, but reversing one coordinate in each of the trivialization isomorphisms $\tau_{M} \oplus \nu \cong m$ and $\eta \oplus \nu \cong p$.

Definition 6. An $l$-trivial manifold $M$ is $l$-trivial cobordant to 0 if there is an $l$-trivial manifold $P$ with $\delta P=M$. Two $l$-trivial manifolds $M$ and $N$ are $l$-trivial cobordant if $M \amalg(-N)$ is $l$-trivial cobordant to 0 .

Thus, we have the cobordism groups of $l$-trivial manifolds for each given $l$.

Let us also establish here some notations and recall certain basic notions in equivariant stable homotopy theory, such as can be found in Lewis, May, and Steinberger [16]. For a $\mathbb{Z} / 2$-equivariant spectrum $E$, we denote homology and cohomology in integral dimensions by $E_{*}, E^{*}$; and we denote homology and cohomology over the complete universe, i.e. all dimensions $k+l \alpha, k, l \in$ $\mathbb{Z}$, by $E_{\star}, E^{\star}$.

Recall the contractible free $\mathbb{Z} / 2$-space $E \mathbb{Z} / 2$, which can be considered as

$$
S(\infty \alpha)=\underline{\lim } S(n \alpha)
$$


where $S(n \alpha)$ is the $n$-dimensional sphere on which $\mathbb{Z} / 2$ acts by the negative map. There is the cofibration sequence

$$
E \mathbb{Z} / 2_{+} \rightarrow S^{0} \rightarrow \widetilde{E \mathbb{Z} / 2} .
$$

The cofiber $E \mathbb{Z} / 2$ is the unreduced suspension of $E \mathbb{Z} / 2$, and is therefore $S^{\infty \alpha}$, the one-point compactification of $\mathbb{R}^{\infty \alpha}$. If $E$ is a $\mathbb{Z} / 2$-equivariant spectrum, smashing the cofibration sequence with $E$ gives the cofiber sequence

$$
E \mathbb{Z} / 2_{+} \wedge E \rightarrow E \rightarrow \widetilde{E \mathbb{Z} / 2} \wedge E .
$$

We can take $\mathbb{Z} / 2$-fixed points of the sequence. Recall also that

$$
(\widetilde{E \mathbb{Z} / 2})^{\mathbb{Z} / 2} \wedge E \simeq\left(S^{\infty \alpha} \wedge E\right)^{\mathbb{Z} / 2}
$$

is the geometric fixed point spectrum of $E$, denoted by $\Phi^{\mathbb{Z} / 2} E$.

Following Atiyah [4] and Landweber [15], let $B \mathbb{U}(n)$ denote the space of $n$-dimensional subspaces of $\mathbb{C}^{\infty}$, with an involution given by complex conjugation. There is a canonical Real bundle $\gamma_{\text {Real }}^{n}$ of dimension $n(1+\alpha)$ over $B \mathbb{U}(n)$, which classifies the Real bundles of dimension $n(1+\alpha)$. We have a map of Thom spaces

$$
\Sigma^{1+\alpha} T\left(\gamma_{\text {Real }}^{n}\right) \rightarrow T\left(\gamma_{\text {Real }}^{n+1}\right) .
$$

Let $M \mathbb{R}$ be the resulting $\mathbb{Z} / 2$-equivariant spectrum. Then $M \mathbb{R}$ is Real oriented. Also, $B \mathbb{U}(n) \times E \mathbb{Z} / 2$ is a free $\mathbb{Z} / 2$-space with $\mathbb{Z} / 2$ acting diagonally. There is the canonical free Real line bundle $\gamma^{n}$ free (of dimension $n(1+\alpha)$ ) over $B \mathbb{U}(n) \times E \mathbb{Z} / 2$, which is the induced bundle of the projection map $B \mathbb{U}(n) \times E \mathbb{Z} / 2 \rightarrow B \mathbb{U}(n)$. There is a prespectrum given by

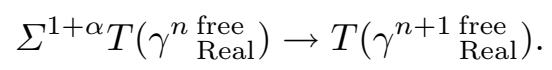

This is $M \mathbb{R} \wedge E \mathbb{Z} / 2_{+}$after spectrification.

3. Free Real cobordism. This section will be dedicated to the proof of Proposition 1, which will be done in a manner analogous to the case of complex cobordism, as shown in Milnor [17] or Milnor and Stasheff [18]. We will show that given any equivariant map from $S^{p+m \alpha}$ to the Thom space $T$ of a smooth Real bundle over a free Real manifold, there is an (equivariant) homotopic smooth map transverse to the zero section of the base manifold. The cobordism of the resulting Real manifold depends only upon the homotopy class of maps, so we have a well defined map from $\pi_{k+l \alpha+n(1+\alpha)}(T)$ to the cobordism group of Real manifolds of dimension $k+l \alpha$.

For any $x$ in a $\mathbb{Z} / 2$-space, write $\bar{x}$ for its image under the action of the nontrivial element of $\mathbb{Z} / 2$. Let $M$ be a compact Real manifold. For $n$ large enough, we can consider $M$ as embedded equivariantly in $\mathbb{R}^{m+p \alpha}=$ $\mathbb{R}^{k+l \alpha+n(1+\alpha)}$, with Real normal bundle $\nu$ of dimension $n(1+\alpha)$. 
LEMma 7. Let $M$ be a compact Real manifold, possibly with boundary, embedded in $\mathbb{R}^{m+p \alpha}$. Then there is an open neighborhood $U$ of $M$ in $\mathbb{R}^{m+p \alpha}$ which is diffeomorphic to the total space $E(\nu)$ of the normal bundle by an equivariant diffeomorphism $\varphi$ that sends each $x \in M$ to the zero normal vector at $x$ in $E(\nu)$.

Proof. Similar to that for the nonequivariant tubular neighborhood lemma.

Let $N, M$ be free Real manifolds, $\xi$ a smooth Real bundle of dimension $n(1+\alpha)$ over $M$, with total space $E(\xi)$ and Thom space $T(\xi)$. Let $\infty \in T(\xi)$ denote the point at infinity. First, we will show that each continuous function $S^{m+p \alpha} \rightarrow T(\xi)$ is homotopic to a map $g$ smooth throughout $g^{-1}(T(\xi)-\infty)=$ $f^{-1}(T(\xi)-\infty)$.

LEMma 8. If $f: N \rightarrow T(\xi)$ is equivariant and continuous, then $f$ is homotopic to a map $g$ which is smooth on $g^{-1}(T(\xi)-\infty)=f^{-1}(T(\xi)-\infty)$.

Proof. Let || be an equivariant Euclidean norm on $E(\xi)$, and $D(\xi)$ be the elements $v \in E(\xi)$ with $|v| \leq 1$. Then $D(\xi)$ is a compact manifold with boundary and a smooth $\mathbb{Z} / 2$-action, and for some $m, p, D(\xi)$ can be smoothly and equivariantly embedded in $\mathbb{R}^{m-1+p \alpha}$ by an embedding $\varphi$. Also, let $\theta:[0,1] \rightarrow[0,1]$ be a smooth map which is strictly increasing and bijective. Then define

$$
\psi: T(\xi) \rightarrow \mathbb{R}^{m+p \alpha}
$$

by

$$
v \mapsto(\theta(|v|),(1-\theta(|v|)) \varphi(v))
$$

for $v \in T(\xi)-\infty=D(\xi)-\delta D(\xi)$, and

$$
\infty \mapsto(1,0, \ldots, 0)
$$

Then $\psi$ gives an embedding of $T(\xi)$ topologically in $\mathbb{R}^{m+p \alpha}$ such that the manifold $T(\xi)-\infty$ is smoothly embedded. By a similar method, $T(\xi)-\infty$ has a tubular neighborhood $U$ diffeomorphic to $E(\nu)$, where $\nu$ is the normal bundle of $T(\xi)-\infty$ in $\mathbb{R}^{m+p \alpha}$.

Let $\delta: N \rightarrow \mathbb{R}$ be a positive function invariant under the $\mathbb{Z} / 2$-action such that $\delta(x) \rightarrow 0$ as $x \rightarrow f^{-1}(\infty)$. Let $U=f^{-1}(T(\xi)-\infty)$. Then by an argument similar to that for the nonequivariant case, we have a smooth $g: U \rightarrow T(\xi)-\infty$ homotopic to $f$ by some $H(x, t)$ such that for all $0 \leq t \leq 1$, $|H(x, t)-f(x)|<\delta$. Then we can extend $H$ by setting $H(x, t)=\infty$ for all $0 \leq t \leq 1, x \in f^{-1}(\infty)$, and $H, g$ will be continuous everywhere. Moreover, $H(x, t)=\infty$ iff $f(x)=\infty$.

The next step shows that for $M$ free, a map $S^{m+p \alpha} \rightarrow T(\xi)$ is homotopic to one that is transverse to the 0 -section $M \subset T(\xi)$. 
Lemma 9. If $M$ is a free Real manifold, $\xi$ is a Real bundle on $M$, and $f$ : $S^{m+p \alpha} \rightarrow T(\xi)$ is equivariant and continuous, then $f$ is homotopic to a map $g$ which is smooth on $f^{-1}(T(\xi)-\infty)$, and transverse to the zero section $M$. The Real cobordism class of the manifold $g^{-1}(M)$ of codimension $n(1+\alpha)$ depends only on the homotopy class of $g$. Thus, we have a well defined map from $\pi_{m+p \alpha}(T(\xi), \infty)$ to the Real cobordism group of Real manifolds of dimension $m+p \alpha-n(1+\alpha)$.

To prove this lemma, we use the following standard lemma (MilnorStasheff [18]).

Lemma 10. Let $U \subseteq \mathbb{C}^{r}$ be open, and $f: U \rightarrow \mathbb{C}^{s}$ smooth, with the origin as a regular value throughout a relatively closed subset $X \subseteq U$. Let $K$ be a compact subset of $U$. Then there is a smooth map $g: U \rightarrow \mathbb{C}^{r}$, $g=f$ outside of a compact subset $K^{\prime} \subseteq U$, and having the origin as a regular value throughout $X \cup K$. Given any $\varepsilon>0$, we can choose $g$ such that $|f(x)-g(x)|<\varepsilon$ for all $x \in U$.

Proof of Lemma 9. By the equivariant smooth approximation lemma, we can assume that $f$ is smooth on $f^{-1}(T(\xi)-\infty)$. Since $M$ is free under the action of $\mathbb{Z} / 2$, so are $E(\xi)$ and $T(\xi)-\infty$. Thus, the equivariant map $f: S^{m+p \alpha} \rightarrow T(\xi)$ must take every fixed point to $\infty$. We can cover the compact set $f^{-1}(M)$ in $S^{p+m \alpha}$ by a finite number of open sets $U_{1}, \ldots, U_{k}$ in $f^{-1}(T(\xi)-\infty)$. Each $U_{i}$ is small enough such that it is disjoint from $\bar{U}_{i}$, where $\bar{U}_{i}$ denotes the image of $U_{i}$ under the action of $\mathbb{Z} / 2$. We also make sure that each $f\left(U_{i}\right) \subseteq T(\xi)-\infty \subseteq E(\xi)$ is contained in some $\xi^{-1}\left(V_{i}\right) \cong V_{i} \times \mathbb{C}^{n}$, where $V_{i}$ is an open coordinate neighborhood of the bundle in $M$. Let $K_{i} \subseteq U_{i}$ be compact, with $f^{-1}(M)$ contained in the interior of $K=\bigcup_{i=1}^{k} K_{i}$. Also, for $x \in T(\xi)-\infty$, we have the Euclidean norm $0 \leq|x|<1$, with $|x|=0$ iff $x \in M$. By compactness, there is a constant $\varepsilon>0$ such that $|f(t)| \geq \varepsilon$ for all $t \notin K$.

Now we proceed according to the standard argument, by constructing a sequence of equivariant maps $f_{0}, f_{1}, \ldots, f_{n}$ such that $f_{0}=f$, each $f_{i}$ is smooth throughout $f_{i}^{-1}(T(\xi)-\infty)=f^{-1}(T(\xi)-\infty)$, and coincides with $f_{i-1}$ outside of a compact subset of $U_{i} \cup \bar{U}_{i}$. Also, we want each $f_{i}$ to be transverse to $M$ throughout $\bigcup_{j=1}^{i}\left(K_{i} \cup \bar{K}_{i}\right)$, and that $\xi\left(f_{i}(x)\right) \in M$ is equal to $\xi(f(x))$ for all $x \in f^{-1}(T(\xi)-\infty)$, i.e. each $f_{i}$ differs from $f_{i-1}$ only within each fiber.

Suppose we have $f_{i-1}$ satisfying these conditions. Then $f_{i-1}$ maps $U_{i}$ into $\xi^{-1}\left(V_{i}\right)$ and $\bar{U}_{i}$ into $\xi^{-1}\left(\bar{V}_{i}\right)$. By the equivariance of $\xi$, we have

$$
\xi^{-1}\left(V_{i}\right) \cong V_{i} \times \mathbb{C}^{n}, \quad \xi^{-1}\left(\bar{V}_{i}\right) \cong \bar{V}_{i} \times \mathbb{C}^{n} .
$$

Let $p_{i}: \xi^{-1}\left(V_{i}\right) \rightarrow \mathbb{C}^{n}$ and $\bar{p}_{i}: \xi^{-1}\left(\bar{V}_{i}\right) \rightarrow \mathbb{C}^{n}$ be the projections onto the second coordinate. Then the composition $p_{i} f_{i-1}$ has the origin as a regular 
value throughout the relatively closed subset $U_{i} \cap \bigcup_{j=1}^{i-1} K_{j}$ in $U_{i}$. Then by Lemma 10, it is approximated by $q_{i}: U_{i} \rightarrow \mathbb{C}^{n}$ which has the origin as a regular value throughout $U_{i} \cap\left(K_{1} \cup \ldots \cup K_{i}\right)$ and differs from $p_{i} f_{i-1}$ only in a compact subset $K_{i}^{\prime}$ of $U_{i}$. We can make it so that

$$
\left|q_{i}(t)-p_{i}\left(f_{i-1}(t)\right)\right|<\varepsilon / k
$$

for all $t$. Define $p_{i} f_{i}: \xi^{-1}\left(V_{i}\right) \rightarrow \mathbb{C}^{n}$ to be $q_{i}$, and $\bar{p}_{i} f_{i}: \xi^{-1}\left(\bar{V}_{i}\right) \rightarrow \mathbb{C}^{n}$ to be the conjugate of $q_{i}$. Since $f_{i}$ and $f_{i-1}$ differ only within fibers of $\xi$, this determines $f_{i}$ on $U_{i} \cup \bar{U}_{i}$, and thus on $S^{m+p \alpha}$, completely. Because we made the corresponding changes over $U_{i}$ and $\bar{U}_{i}, f_{i}$ is equivariant. It is easy to check that $f_{i}$ satisfies all the desired conditions. Let $g=f_{k}$. Then $g$ is smooth, equivariant, and transverse to $M$ throughout the compact set $K$.

We chose each $f_{i}$ such that for every $t$,

$$
\left|f_{i}(t)-f_{i-1}(t)\right|<\varepsilon / k .
$$

Then $|g(t)-f(t)|<\varepsilon$ for every $t$, so $|g(t)| \neq 0$, i.e. $g(t) \notin M$ for every $t \notin K$. Hence, we have $g^{-1}(M) \subseteq K$. Therefore, $g$ is transverse to $M$ throughout $S^{m+p \alpha}$, and $g^{-1}(M)$ is a manifold with a Real structure induced by $g$.

If $g$ and $g^{\prime}$ are two maps $S^{m+p \alpha} \rightarrow T(\xi)$, smooth throughout the inverse images of $T(\xi)-\infty$ and transverse to $M$, and homotopic by $h$, then we can make the homotopy $h$ transverse to $M$. Thus, the inverse image $h^{-1}(X)$ is a Real manifold with boundary, giving the cobordism between $g^{-1}(X)$ and $g^{\prime-1}(X)$. Hence, we have a well defined map from $\pi_{m+p \alpha}(T(\xi))$ to the $(m+p \alpha-n(1+\alpha))$ th cobordism group of free Real manifolds. It is easy to see that this is a group homomorphism, just as in the case of real and complex manifolds.

We are now ready to prove Proposition 1, which gives the free Real cobordism groups of free Real manifolds. We shall denote these cobordism groups by $\Omega_{\text {free }}^{\text {Real }}(k+l \alpha)$.

Proof of Proposition 1. Let $\mathbb{G}_{n, t}$ be the space of all $n$-dimensional subspaces in $\mathbb{C}^{t}$, with $\mathbb{Z} / 2$-action by conjugation. Then $\mathbb{G}_{n, t}$ has the structure of a Real manifold, and

$$
B \mathbb{U}(n)=\bigcup_{t} \mathbb{G}_{n, t} .
$$

There is a canonical Real line bundle $\gamma^{n, t}$ over $\mathbb{G}_{n, t}$, of dimension $n(1+\alpha)$, and for $t$ large enough, $\gamma^{n, t}$ classifies Real bundles of dimension $n(1+\alpha)$ over paracompact spaces. The projection

$$
\mathbb{G}_{n, t} \times E \mathbb{Z} / 2_{+} \rightarrow \mathbb{G}_{n, t}
$$

induces the bundle $\gamma^{n, t}$ Real over $\mathbb{G}_{n, t}$, which classifies Real bundles of dimension $n(1+\alpha)$ over free $\mathbb{Z} / 2$-spaces. 
Let $M$ be a compact free Real manifold of dimension $k+l \alpha$. Then for $n, t$ large enough, $M$ is embedded equivariantly in $\mathbb{R}^{k+l \alpha+n(1+\alpha)}$, with normal bundle $\nu^{n}$. There is the canonical smooth equivariant map

$$
\varphi: M \rightarrow \mathbb{G}_{n, t} \times E \mathbb{Z} / 2
$$

where the first coordinate is the classification map of $\nu^{n}$. The second coordinate is the canonical map from a free space to $E \mathbb{Z} / 2=S(\infty \alpha)$. For some $s \geq 0, M$ is mapped into the finite skeleton $S(s \alpha)$ of $E \mathbb{Z} / 2$. So we have

$$
M \stackrel{\varphi}{\rightarrow} \mathbb{G}_{n, t} \times S(s \alpha) \stackrel{i}{\rightarrow} \mathbb{G}_{n, t} \times E \mathbb{Z} / 2 .
$$

Now the inclusion $i$ induces the bundle $\gamma_{s}^{n, t}$ Real over $\mathbb{G}_{n, t} \times E \mathbb{Z} / 2$, which has the structure of a compact free Real manifold. $\varphi$ gives a map of Real bundles $\nu \rightarrow \gamma_{s, \text { free }}^{n, t}$. Thus by the tubular neighborhood lemma, we have

$$
\varphi: U \cong E(\nu) \rightarrow E\left(\gamma_{s}^{n, t} \text { Real } \text { Rree }\right) \rightarrow T\left(\gamma_{s}^{n, t} \text { Rree } \text { Real }\right)
$$

which we can extend to $S^{m+p \alpha}$, the one-point compactification of $\mathbb{R}^{m+p \alpha}$, by mapping $S^{m+p \alpha}-U$ to $\infty$. This is an equivariant smooth map transverse to the zero section $B \mathbb{U}(n) \times E \mathbb{Z} / 2$ of $\gamma_{\text {Real }}^{n \text { free }}$, and

$$
\varphi^{-1}\left(\mathbb{G}_{n, t} \times S(s \alpha)\right)=M .
$$

Thus, $M$ is in the image of the map

$$
\pi_{k+l \alpha+n(1+\alpha)}\left(T\left(\gamma_{s}^{n, t} \text { Real }\right)\right) \rightarrow \Omega_{\text {free }}^{\text {Real }}(k+l \alpha) .
$$

Passing to the limit over $s$, we get the surjective map

$$
\pi_{k+l \alpha+n(1+\alpha)}\left(T\left(\gamma^{n, t} \underset{\text { Real }}{\text { free }}\right)\right) \rightarrow \Omega_{\text {freal }}^{\text {Real }}(k+l \alpha) .
$$

Injectivity is proved in a similar manner for $n, t$ sufficiently large. Thus, we have

$$
\pi_{k+l \alpha+n(1+\alpha)}\left(T\left(\gamma_{\text {Real }}^{n, t \text { free }}\right)\right) \cong \Omega_{\text {free }}^{\text {Real }}(k+l \alpha) .
$$

This shows that $\Omega_{\text {Real }}^{\text {free }}(k+l \alpha)$ is isomorphic to the stable homotopy group $\pi_{k+l \alpha}\left(M \mathbb{R} \wedge E \mathbb{Z} / 2_{+}\right)$, which is our theorem.

4. Cobordism of general Real manifolds. Let $M$ be a compact Real manifold of dimension $k+l \alpha$, not necessarily free. Let $M^{\mathbb{Z} / 2}$ denote the subspace of $M$ fixed by the action of $\mathbb{Z} / 2$. Then $M^{\mathbb{Z} / 2}$ is a compact real submanifold of $M$.

Lemma 11. The dimension of $M^{\mathbb{Z} / 2}$ is $k$.

Proof. There is a Real normal bundle $\nu$ on $M$ of dimension $n(1+\alpha)$ such that $\tau_{M} \oplus \nu=m+p \alpha$, where $m=k+n, p=l+n$. Restricting to $M^{\mathbb{Z} / 2}$, we have

$$
\left.\left.\tau_{M}\right|_{M^{\mathbb{Z} / 2}} \oplus \nu\right|_{M^{\mathbb{Z} / 2}}=m+\left.p \alpha\right|_{M^{\mathbb{Z} / 2}} .
$$


Since $M^{\mathbb{Z} / 2}$ is fixed, the $\mathbb{Z} / 2$-action on a bundle over it takes each fiber onto itself, so we can consider its fixed subbundle. The Real structure of $\nu$ gives that over $M^{\mathbb{Z} / 2}$, the $\mathbb{Z} / 2$-action on each fiber is a complex antilinear transformation. Hence, each fiber, of complex dimension $n$, is the direct sum of a fixed real subspace, and a real subspace where the $\mathbb{Z} / 2$-action is multiplication by -1 , where each subspace has dimension $n$. Let $\nu^{\mathbb{Z} / 2}$ denote the fixed subbundle of $\nu$. Then $\nu^{\mathbb{Z} / 2}$ has real dimension $n$, and $\nu$ breaks into two copies of $\nu^{\mathbb{Z} / 2}$, i.e.

$$
\nu=\nu^{\mathbb{Z} / 2}(1+\alpha)=\nu^{\mathbb{Z} / 2} \oplus i \nu^{\mathbb{Z} / 2}
$$

where the $\mathbb{Z} / 2$-action is complex conjugation. Also, at each point $x \in M^{\mathbb{Z} / 2}$, $M$ is locally equivariantly diffeomorphic to a representation of $\mathbb{Z} / 2$. Thus, the fixed subbundle of $\left.\tau_{M}\right|_{M^{\mathbb{Z} / 2}}$ is $\tau_{M^{\mathbb{Z} / 2}}$. Finally, the fixed subbundle of $m+p \alpha$ is $m$. Thus, taking $\mathbb{Z} / 2$-fixed points of the earlier equation, we get

$$
\tau_{M / 2}^{\mathbb{Z}} \oplus \nu^{\mathbb{Z} / 2}=m \text {. }
$$

So the dimension of $M^{\mathbb{Z} / 2}$ is $m-n=k$.

In particular, this shows that if a Real manifold $M$ has a nonempty fixed submanifold $M^{\mathbb{Z} / 2}$, then the Real dimension $k+l \alpha$ of $M$ is independent of the Real normal bundle giving $M$ its Real structure, and that $k, l \geq 0$.

What can we say about the fixed submanifold $M^{\mathbb{Z} / 2}$ ? Note that there is the normal bundle $\nu_{M^{\mathbb{Z} / 2}}^{M}$ of $M^{\mathbb{Z} / 2}$ in $M$, with

$$
m+p \alpha=\left.\left.\tau_{M}\right|_{M^{\mathbb{Z} / 2}} \oplus \nu\right|_{M^{\mathbb{Z} / 2}}=\tau_{M^{\mathbb{Z} / 2}} \oplus \nu_{M^{\mathbb{Z} / 2}}^{M} \oplus \nu^{\mathbb{Z} / 2}(1+\alpha) .
$$

By the previous lemma, we have $\tau_{M^{\mathbb{Z} / 2}} \oplus \nu^{\mathbb{Z} / 2}=m$. Also, taking the nonfixed complements, we get $\nu_{M^{\mathbb{Z} / 2}}^{M} \oplus \nu^{\mathbb{Z} / 2} \alpha=p \alpha$. In particular, $\nu_{M^{\mathbb{Z} / 2}}^{M}$ has dimension $l \alpha$.

Considering only the real structures of the bundles, we see that a normal bundle $\nu^{\mathbb{Z} / 2}$ of $M^{\mathbb{Z} / 2}$ is trivialized by an $l$-dimensional bundle. Thus, for a Real manifold $M$ of dimension $k+l \alpha, M^{\mathbb{Z} / 2}$ is an $l$-trivial real manifold.

We now prove Proposition 2, which determines the cobordism spectrum of compact $l$-trivial manifolds.

Proof of Proposition 2. The classifying space for sums of pairs of bundles $\xi \oplus \eta$ over a space $X$, with $|\xi|=n,|\eta|=l$, is $B O(n) \times B O(l)$, and the universal bundle is $\gamma^{n} \times \gamma^{l}$. There is a map

$$
\theta: B O(n) \times B O(l) \rightarrow B O(n+l)
$$

that classifies $\gamma^{n} \times \gamma^{l}$. Thus, if $\xi \oplus \eta$ is trivial, the map $X \rightarrow B O(n) \times B O(l)$, classifying the sum $\xi \oplus \eta$ over $X$, pulls back to the homotopy fiber of $\theta$, which is $O(n+l) /(O(n) \times O(l))$. 
The universal $n$-dimensional $l$-trivial bundle, which we denote by $\bar{\gamma}_{l}^{n}$, is the pullback of $\gamma^{n}$ over $B O(n)$ under the map

$$
O(n+l) /(O(n) \times O(l)) \rightarrow B O(n) \times B O(l) \rightarrow B O(n) .
$$

By the usual procedure, the cobordism of $l$-trivial manifolds is given by the Thom spectrum $\lim _{n \rightarrow \infty} \Sigma^{-n} T\left(\bar{\gamma}_{l}^{n}\right)$. As $n \rightarrow \infty, O(n) \rightarrow O(n+l)$, so

$$
O(n+l) /(O(n) \times O(l)) \rightarrow B O(l) .
$$

Hence

$$
\lim _{n \rightarrow \infty} \Sigma^{-n} T\left(\bar{\gamma}_{l}^{n}\right)=\Sigma^{-l} T\left(\left.\gamma^{l}\right|_{B O(l)}\right) .
$$

Let $\bar{\Omega}^{l}(k)$ denote the cobordism group of compact $k$-dimensional $l$-trivial manifolds. Then

$$
\pi_{k}\left(\Sigma^{-l} T\left(\left.\gamma^{l}\right|_{B O(l)}\right)\right) \cong \bar{\Omega}^{l}(k),
$$

i.e. $\Sigma^{-l} T\left(\left.\gamma^{l}\right|_{B O(l)}\right)$ is the cobordism spectrum of compact $l$-trivial manifolds.

We are now ready to prove Theorem 3. First, consider the manifolds represented by $\Sigma^{-l} T\left(\left.\gamma^{l}\right|_{B O(l)}\right)$. Suppose that we have a $k$-dimensional compact $l$-trivial manifold $N$, with normal bundle $\nu$ and $l$-dimensional bundle $\eta$, such that $\tau_{N} \oplus \nu=m, \eta \oplus \nu=p$. Let || be a Riemannian metric on $\nu$, and

$$
D(\eta)=\left\{(x, v)\left|x \in N, v \in \eta_{x},\right| v \mid \leq 1\right\}
$$

be the disk bundle of $\nu$ with respect to | |. Give an action of $\mathbb{Z} / 2$ on $D(\eta)$ by

$$
(x, v) \mapsto(x,-v) .
$$

Then $D(\eta)$ is a real manifold with boundary

$$
S(\eta)=\left\{(x, v)\left|x \in N, v \in \eta_{x},\right| v \mid=1\right\} .
$$

$D(\eta)$ has a smooth $\mathbb{Z} / 2$-action that sends $S(\eta)$ to $S(\eta)$, and its tangent bundle is

$$
\tau_{D(\eta)}=\tau_{N} \oplus \eta
$$

with $\mathbb{Z} / 2$ fixing $\tau_{N}$ and acting by -1 on $\eta$. The bundle $\nu \oplus i \nu$ on $N$, with $\mathbb{Z} / 2$-action given by complex conjugation, has a natural Real structure. Let $\nu(1+\alpha)$ be the pullback of $\nu \oplus i \nu$ under the projection map $D(\eta) \rightarrow N$. Then there is an induced Real structure on $\nu(1+\alpha)$. Note that

$$
\tau_{D(\eta)} \oplus \nu(1+\alpha)=\tau_{N} \oplus \eta \oplus \nu(1+\alpha)=m+p \alpha .
$$

Also, the collar bundle of $S(\eta)$ in $D(\eta)$ is fixed under the $\mathbb{Z} / 2$-action. Hence, $D(\eta)$ is a Real manifold with boundary, of dimension $k+l \alpha$, so $S(\eta)$ is a free Real manifold of dimension $(k-1)+l \alpha$, with the Real normal bundle $\nu(1+\alpha)$. If $\bar{N}$ is an $l$-trivial manifold with boundary $N$ and $l$-dimensional 
bundle $\bar{\eta}$, then $S\left(\left.\bar{\eta}\right|_{N}\right)$ is the boundary of $S(\bar{\eta})$. Thus, $(N, \eta) \mapsto S(\eta)$ is a well defined map $\bar{\Omega}^{l}(k) \rightarrow \Omega_{\text {free }}^{\text {Real }}(k-1+l \alpha)$.

Now recall the connecting map (2):

$$
\delta: \Sigma^{-l} T\left(\left.\gamma^{l}\right|_{B O(l)}\right) \rightarrow M O \rightarrow \Sigma\left(\Sigma^{-l \alpha}\left(M \mathbb{R} \wedge E \mathbb{Z} / 2_{+}\right)^{\mathbb{Z} / 2}\right)
$$

defined by means of homotopy theory. On coefficients, $\delta$ gives the map

$$
\begin{aligned}
\delta_{*}: \bar{\Omega}^{l}(k) & =\pi_{k}\left(\Sigma^{-l} T\left(\left.\gamma^{l}\right|_{B O(l)}\right)\right) \\
& \rightarrow \pi_{k}\left(\Sigma\left(\Sigma^{-l \alpha}\left(M \mathbb{R} \wedge E \mathbb{Z} / 2_{+}\right)^{\mathbb{Z} / 2}\right)\right)=\pi_{k-1+l \alpha}\left(M \mathbb{R} \wedge E \mathbb{Z} / 2_{+}\right) \\
& =\Omega_{\text {free }}^{\text {Real }}(k-1+l \alpha) .
\end{aligned}
$$

LEMMA 12. $\delta_{*}: \bar{\Omega}^{l}(k) \rightarrow \Omega_{\text {free }}^{\text {Real }}(k-1+l \alpha)$ sends the cobordism class of $N$, with l-dimensional bundle $\eta$ and normal bundle $\nu$, to the class of $S(\eta)$ with a Real normal bundle $\nu(1+\alpha)$.

Proof. $\Sigma^{-l} T\left(\left.\gamma^{l}\right|_{B O(l)}\right)$ is the Thom spectrum of the pullback of $\gamma^{n}$ over $O(n+l) /(O(n) \times O(l))$, and the stabilization map $\iota: \Sigma^{-l} T\left(\left.\gamma^{l}\right|_{B O(l)}\right) \rightarrow M O$ is

$$
\iota: T\left(\left.\gamma^{n}\right|_{O(n+l) /(O(n) \times O(l))}\right) \rightarrow T\left(\left.\gamma^{n}\right|_{B O(n)}\right)
$$

on the prespectrum level.

Let $S\left(\gamma^{l} \alpha\right)$ be the sphere bundle of $\gamma^{l} \alpha$ over $B O(l)$, and let $S^{\gamma^{l} \alpha}$ be its unreduced suspension. Then there is a map

$$
T\left(\left.\gamma^{l} \alpha\right|_{B O(l)}\right) \rightarrow S^{\gamma^{l} \alpha}
$$

given by contracting the 0 -section to a single point. Also, there is a classification map $S\left(\gamma^{l} \alpha\right)_{+} \rightarrow S(\infty \alpha)_{+}=E \mathbb{Z} / 2_{+}$, giving a map

$$
c: S^{\gamma^{l} \alpha} \rightarrow S^{\infty \alpha} \text {. }
$$

Moreover, $\gamma^{n}(1+\alpha)$ is Real of dimension $n(1+\alpha)$ over the fixed space $B O(n)$, so there is a classification map

$$
\left.\left.\gamma^{n}(1+\alpha)\right|_{B O(n)} \rightarrow \gamma_{\text {Real }}^{n}\right|_{B \mathbb{U}(n)} .
$$

Hence, we have the map of Thom spaces

$$
\begin{aligned}
\Sigma^{(n+l) \alpha} T\left(\left.\gamma^{n}\right|_{O(n+l) /(O(n) \times O(l))}\right) & \cong T\left(\left.\gamma^{n}(1+\alpha) \oplus \gamma^{l} \alpha\right|_{O(n+l) /(O(n) \times O(l))}\right) \\
& \stackrel{\iota}{\rightarrow} T\left(\gamma^{n}(1+\alpha) \times\left.\gamma^{l} \alpha\right|_{B O(n) \times B O(l)}\right) \\
& \cong T\left(\left.\gamma^{n}(1+\alpha)\right|_{B O(n)}\right) \wedge T\left(\left.\gamma^{l} \alpha\right|_{B O(l)}\right) \\
& \rightarrow T\left(\left.\gamma_{\text {Real }}^{n}\right|_{B \mathbb{U}(n)}\right) \wedge S^{\gamma^{l} \alpha} \\
& \stackrel{c}{\rightarrow} T\left(\left.\gamma_{\text {Real }}^{n}\right|_{B \mathbb{U}(n)}\right) \wedge S^{\infty \alpha} \\
& \stackrel{s}{\rightarrow} \Sigma^{l \alpha} T\left(\left.\gamma_{\text {Real }}^{n}\right|_{B \mathbb{U}(n)}\right) \wedge S^{\infty \alpha} \\
& \stackrel{d}{\rightarrow} \Sigma^{l \alpha} T\left(\left.\gamma_{\text {Real }}^{n}\right|_{B \mathbb{U}(n)}\right) \wedge \Sigma E \mathbb{Z} / 2_{+} \\
& =\Sigma \Sigma^{l \alpha} T\left(\left.\gamma_{\text {Real }}^{n \text { free }}\right|_{B \mathbb{U}(n) \times E \mathbb{Z} / 2}\right)
\end{aligned}
$$


where the map

$$
s: T\left(\left.\gamma_{\text {Real }}^{n}\right|_{B \mathbb{U}(n)}\right) \wedge S^{\infty \alpha} \rightarrow \Sigma^{-l \alpha} T\left(\left.\gamma_{\text {Real }}^{n}\right|_{B \mathbb{U}(n)}\right) \wedge S^{\infty \alpha}
$$

is inverse to the map induced by the suspension $S^{0} \rightarrow S^{l \alpha}$. Since $\Sigma^{l \alpha} S^{\infty \alpha} \cong$ $S^{\infty \alpha}, s$ is a homeomorphism. Passing to spectra, this gives the map of $\mathbb{Z} / 2$ spectra (with $\Sigma^{-l} T\left(\left.\gamma^{l}\right|_{B O(l)}\right)$ considered as fixed):

$$
\Sigma^{-l} T\left(\left.\gamma^{l}\right|_{B O(l)}\right) \rightarrow \Sigma^{-l \alpha}\left(M \mathbb{R} \wedge S^{\infty \alpha}\right) \stackrel{d}{\rightarrow} \Sigma\left(\Sigma^{-l \alpha}\left(M \mathbb{R} \wedge E \mathbb{Z} / 2_{+}\right)\right) .
$$

Taking $\mathbb{Z} / 2$-fixed points, it becomes our map

$$
\delta: \Sigma^{-l} T\left(\left.\gamma^{l}\right|_{B O(l)}\right) \stackrel{\iota}{\rightarrow} M O \stackrel{d}{\rightarrow} \Sigma\left(\Sigma^{-l \alpha}\left(M \mathbb{R} \wedge E \mathbb{Z} / 2_{+}\right)^{\mathbb{Z} / 2}\right) .
$$

Since the diagram

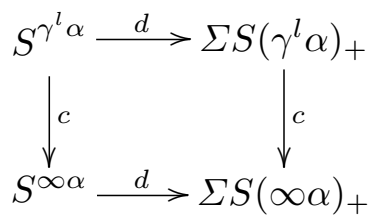

is commutative, the above map of Thom spaces is the same as

$$
\begin{aligned}
\Sigma^{(n+l) \alpha} T\left(\left.\gamma^{n}\right|_{O(n+l) /(O(n) \times O(l))}\right) & =T\left(\left.\gamma^{n}(1+\alpha) \oplus \gamma^{l} \alpha\right|_{O(n+l) /(O(n) \times O(l))}\right) \\
& \stackrel{\iota}{\rightarrow} T\left(\left.\gamma^{n}(1+\alpha) \oplus \gamma^{l} \alpha\right|_{B O(n) \times B O(l)}\right) \\
& =T\left(\left.\gamma^{n}(1+\alpha)\right|_{B O(n)}\right) \wedge T\left(\left.\gamma^{l} \alpha\right|_{B O(l)}\right) \\
& \rightarrow T\left(\left.\gamma_{\text {Real }}^{n}\right|_{B \mathbb{U}(n)}\right) \wedge S^{\gamma^{l} \alpha} \\
& \stackrel{d}{\rightarrow} T\left(\left.\gamma_{\text {Real }}^{n}\right|_{B \mathbb{U}(n)}\right) \wedge \Sigma S\left(\gamma^{l} \alpha\right)_{+} \\
& =T\left(\left.\gamma_{\text {Real }}^{n}\right|_{B \mathbb{U}(n)}\right) \wedge T\left(\left.1\right|_{S\left(\gamma^{l} \alpha\right)}\right) \\
& =T\left(\gamma_{\text {Real }}^{n} \times\left. 1\right|_{B \mathbb{U}(n) \times S\left(\gamma^{l} \alpha\right)}\right) \\
& \stackrel{s}{\rightarrow} \Sigma \Sigma^{l \alpha} T\left(\left.\gamma_{\text {Real }}^{n}\right|_{B \mathbb{U}(n) \times S\left(\gamma^{l} \alpha\right)}\right) \\
& \rightarrow \Sigma \Sigma^{l \alpha} T\left(\left.\gamma_{\text {Real }}^{n \text { free }}\right|_{B \mathbb{U}(n) \times E \mathbb{Z} / 2}\right) .
\end{aligned}
$$

Given an $l$-trivial manifold $N$ of dimension $k$, with $l$-dimensional bundle $\eta$ and $n$-dimensional normal bundle $\nu$, for $n \gg 0$ the cobordism class of $N$ is represented by a smooth map

$$
f: S^{k+n} \rightarrow T\left(\gamma^{n} \mid O(n+l) /(O(n) \times O(l))\right)
$$

such that $N$ is the preimage of the 0 -section. We also have

$$
\Sigma^{(n+l) \alpha} f: S^{k+n+(n+l) \alpha} \rightarrow \Sigma^{(n+l) \alpha} T\left(\left.\gamma^{n}\right|_{O(n+l) /(O(n) \times O(l))}\right) .
$$

Following the maps of Thom spaces to $\Sigma T\left(\left.\gamma_{\text {Real }}^{n}\right|_{B \mathbb{U}(n) \times S\left(\gamma^{l} \alpha\right)}\right)$, we see that the 0 -section changes from $O(n+l) /(O(n) \times O(l))$ to $B \mathbb{U}(n) \times S\left(\gamma^{l} \alpha\right)$. Hence, the preimage of the 0 -section changes from $N$ to the universal sphere bundle $S(\eta)$, giving the desired map of cobordism groups. 
The following lemma appears superficially similar to Theorem 1 of Landweber [14]. However, the two are different: Landweber's theorem dealt with cobordism after forgetting the Real structure, whereas we consider the fixed points of a Real manifold.

For a real manifold $M$ with a submanifold $N$, let $\left.\nu\right|_{N} ^{M}$ denote the normal bundle of $N$ in $M$.

Lemma 13. If in a compact Real manifold $M$ of dimension $k+l \alpha, M^{\mathbb{Z} / 2}$ is cobordant to 0 in the l-trivial category, then $M$ is Real cobordant to a free Real manifold $M^{\prime}$.

Proof. Let $M^{\prime}$ be an $l$-trivial manifold with boundary $M^{\mathbb{Z} / 2}$. Then there is an $l$-dimensional bundle $\eta$ on $M^{\prime}$ with

$$
\left.\left.\eta\right|_{M^{\mathbb{Z} / 2}} \cong \nu\right|_{M^{\mathbb{Z} / 2}} ^{M} \text {. }
$$

Let $T$ be a tubular neighborhood of $M^{\mathbb{Z} / 2}$ in $M$ with $T \cong E\left(\left.\nu\right|_{M^{\mathbb{Z} / 2}} ^{M}\right)$. Let $\delta T$ denote the boundary of $T$. Then we can construct $S\left(\left.\eta\right|_{M^{\prime}}\right)$, and attach it to $M$ by identifying

$$
S\left(\left.\nu\right|_{M^{\mathbb{Z} / 2}} ^{M}\right) \cong S\left(\left.\eta\right|_{M^{\mathbb{Z} / 2}}\right)
$$

with $\delta T$. The resulting space $X$ is a smooth manifold except at $\delta T=$ $S\left(\left.\eta\right|_{M^{\mathbb{Z} / 2}}\right)$, with a free $\mathbb{Z} / 2$-action that is smooth except at $\delta T$, so $X-\delta T$ has the structure of a free Real manifold of dimension $k+l \alpha$, except at $\delta T$. Also, let $Y$ be the space obtained by attaching $M \times[0,1]$ to $D(\eta)$ by identifying $D\left(\left.\eta\right|_{M^{\mathbb{Z} / 2}}\right)$ with $\bar{T}$, the closure of $T$. Then $Y$ has the structure of a $(k+1+l \alpha)$-dimensional Real manifold with boundary except at $\delta T$, and the "boundary" of $Y$ is $X \amalg(-M)$. Thus, we will get a free Real manifold cobordant to $M$ if we can "smooth out" the corners of $X$ at $\delta T$.

Since $\delta T$ is compact, there is another tubular neighborhood $S$ of $M^{\mathbb{Z} / 2}$ in $M$,

$$
S \cong E\left(\left.\nu\right|_{M^{\mathbb{Z} / 2}} ^{M}\right)
$$

such that $\bar{T} \subset S$, and there is a collar neighborhood $U \cong \delta S \times[0,1)$ of $\delta S$ in $\bar{S}$ with $\delta T \subset U$. Also,

$$
\delta T \cong S\left(\left.\nu\right|_{M^{\mathbb{Z} / 2}} ^{M} \cong \delta S\right.
$$

so we have

$$
S-T \cong \delta T \times[0,1) .
$$

There is also a collar neighborhood $V \cong S\left(\left.\eta\right|_{M^{\mathbb{Z} / 2}}\right) \times[0,1)$ of $S\left(\left.\eta\right|_{M^{\mathbb{Z} / 2}}\right)$ in $S\left(\left.\eta\right|_{M^{\prime}}\right)$. Hence, we have a neighborhood of $\delta T$ in $X$

$$
(S-T) \cup V \cong(\delta T \times[0,1)) \cup(\delta T \times[0,1)) \cong \delta T \times([0,1) \cup[0,1)) .
$$


We can smooth $(S-T) \cup V$ by smoothing out $[0,1) \cup[0,1)$. More precisely, let $f:(0,1 / 2) \rightarrow(0,1 / 2)$ be a smooth map such that

$$
\lim _{x \rightarrow 0} f(x)=1 / 2, \quad \lim _{x \rightarrow 1 / 2} f(x)=0,
$$

and for all derivatives $f^{(i)}$ of $f$,

$$
\lim _{x \rightarrow 0} f^{(i)}(x)=\infty, \quad \lim _{x \rightarrow 1 / 2} f^{(i)}(x)=0 .
$$

Let

$$
\begin{aligned}
& W_{1}=\{(0, y) \mid 1 / 2 \leq y<1\}, \\
& W_{2}=\{(x, f(x)) \mid 0<x<1 / 2\}, \\
& W_{3}=\{(x, 0) \mid 1 / 2 \leq x<1\} .
\end{aligned}
$$

Let $W=W_{1} \cup W_{2} \cup W_{3}$. Then $W$ is a smooth 1-dimensional manifold. By attaching $\delta T \times W$ to $X-((S-T) \cup V)$, we obtain a smooth manifold $P$ with a free smooth $\mathbb{Z} / 2$-action. Similarly, let

$$
W_{4}=\{(x, y) \mid 0<x<1 / 2,0 \leq y \leq f(x)\} .
$$

Let $U=W_{1} \cup W_{4} \cup W_{3}$. Then, attaching $U$ to $Y$, we get a smooth $\mathbb{Z} / 2$ manifold $Z$ with boundary $P$.

It remains to give a Real normal bundle to $W$ that will be a smooth transition between the Real normal bundles of $M-\bar{S}$ and $S(\eta)-\bar{V}$. Let $\xi^{\prime}$ be the collar bundle of $M^{\mathbb{Z} / 2}$ in $M^{\prime}$, and $\xi$ be the collar bundle of $\delta S$ in $\bar{S}$. Then $\xi, \xi^{\prime}$ are 1-dimensional and trivial. Note

$$
\left.\tau_{M}\right|_{\delta S}=\xi \oplus \tau_{\delta S} \cong \xi \oplus \tau_{\delta T} \quad \text { and }\left.\quad \tau_{S(\eta)}\right|_{\delta V}=\xi^{\prime} \oplus \tau_{\delta T} .
$$

Then $W$ gives a smooth transition between between $\xi$ and $\xi^{\prime}$, i.e.

$$
\tau_{\bar{W}} \cong \mu \oplus \tau_{\delta T}
$$

where $\mu$ is a 1-dimensional trivial real bundle such that

$$
\left.\mu\right|_{\delta S}=\xi,\left.\quad \mu\right|_{\delta V}=\xi^{\prime} .
$$

Let $\nu_{M}$ be a Real normal bundle of $M$ with dimension $n(1+\alpha)$. Then at $\delta S$,

$$
\begin{aligned}
\left.\tau_{M} \oplus \nu_{M}\right|_{\delta S} \oplus \xi^{\prime} \oplus \alpha & \left.\cong \xi \oplus \tau_{\delta S} \oplus \nu_{M}\right|_{\delta S} \oplus \xi^{\prime} \oplus \alpha \\
& \cong(k+n)+(l+n) \alpha \oplus \xi^{\prime} \oplus \alpha \\
& \cong(k+n+1)+(l+n+1) \alpha .
\end{aligned}
$$

Extending $\xi^{\prime}$ to a trivial bundle of dimension 1 on $M$, we see that $\nu_{M} \oplus \xi^{\prime} \oplus \alpha$ is a Real normal bundle to $M$. Also, $\left.\tau_{M^{\prime}}\right|_{\delta V} \cong \xi^{\prime} \oplus \tau_{\delta T}$, so

$$
\left.\left.\tau_{M}\right|_{\delta V} \oplus \nu_{M}\right|_{\delta S} \oplus \xi \oplus \alpha \cong(k+n+1)+(l+n+1) \alpha .
$$

Since $M^{\prime} \cong S\left(\nu_{M^{\mathbb{Z} / 2}}^{M}\right) \times[0,1)$ near $\delta T$, at $\delta V,\left.\nu_{M}\right|_{\delta S} \oplus \xi \oplus \alpha$ extends to a Real normal bundle of $M^{\prime}$. We can find a smooth trivial 1-dimensional bundle $\mu^{\prime}$ 
over $\bar{W}$ such that

$$
\left.\mu^{\prime}\right|_{\delta S} \cong \xi^{\prime},\left.\quad \mu^{\prime}\right|_{\delta V} \cong \xi .
$$

Then $\mu \oplus \mu^{\prime} \cong 2$, and at every $x \in \bar{W}$,

$$
\begin{aligned}
\left.\tau_{\bar{W}} \oplus \nu_{M}\right|_{\delta S} \oplus \mu^{\prime} \oplus \alpha & \left.\cong \mu \oplus \tau_{\delta S} \oplus \nu_{M}\right|_{\delta S} \oplus \mu^{\prime} \oplus \alpha \\
& \left.\cong 2 \oplus \tau_{\delta S} \oplus \nu_{M}\right|_{\delta S} \oplus \alpha \\
& \left.\cong \xi \oplus \tau_{\delta S} \oplus \nu_{M}\right|_{\delta S} \oplus \xi^{\prime} \oplus \alpha \\
& \left.\cong \tau_{M}\right|_{\delta S} \oplus\left(\left.\nu_{M}\right|_{\delta S} \oplus \xi^{\prime} \oplus \alpha\right) \\
& \cong(k+n+1)+(l+n+1) \alpha .
\end{aligned}
$$

So $\left.\nu_{M}\right|_{\delta} \oplus \mu^{\prime} \oplus \alpha$ gives a Real normal bundle on $\bar{W}$ which is a smooth transition between the Real normal bundles of $M$ and $M^{\prime}$. Therefore, $P$ has the structure of a free Real manifold. Similarly, the manifold $Z$ with boundary $P$ has a compatible Real structure, giving a Real cobordism between $P$ and the original manifold $M$.

Proof of Theorem 3. We have the long exact sequence of homotopy groups

$$
\begin{aligned}
\ldots \rightarrow \pi_{k}\left(M \mathbb{R}_{l}\right) \stackrel{p}{\rightarrow} \pi_{k}\left(\Sigma^{-l} T\left(\left.\gamma^{l}\right|_{B O(l)}\right)\right) \\
\qquad \stackrel{\delta_{*}}{\rightarrow} \pi_{k-1+l \alpha}\left(M \mathbb{R} \wedge E \mathbb{Z} / 2_{+}\right) \rightarrow \ldots
\end{aligned}
$$

Given a class $[f]$ in $\pi_{k}\left(M \mathbb{R}_{l}\right),[p(f)]$ determines a cobordism class in the $l$-trivial category, represented by the manifold $N$, with $l$-dimensional bundle $\eta$ and real normal bundle $\nu$. Also, $f$ gives a homotopy from $\delta_{*} p(f)$ to 0 , which gives a free Real cobordism $M^{\prime}$ of $S(\eta)$ to 0 . Thus, we can combine $D(\eta)$ and $M^{\prime}$ along their common boundary $S(\eta)$, giving a Real manifold $M$ of dimension $k+l \alpha$, with the Real normal bundle $\nu(1+\alpha)$. Thus, define the map

$$
\varphi: \pi_{k}\left(M \mathbb{R}_{l}\right) \rightarrow \Omega^{\text {Real }}(k+l \alpha)
$$

by letting $\varphi[f]$ be the cobordism class of $M$. The cobordism class of $N$ depends only on the homotopy class of $f$. Suppose that $\bar{N}$ is a $(k+1)$ dimensional $l$-trivial manifold with boundary $N$, with normal bundle $\bar{\nu}$ and $l$-dimensional real bundle $\bar{\eta}$. Then $N^{\prime}$ gives a smooth homotopy $H$ of $p(f)$ to 0 . Also, there is a smooth homotopy of $\delta_{*} H$ to 0 , giving the space $\bar{M}^{\prime}$. We can combine $D(\bar{\eta})$ and $\bar{M}^{\prime}$, which gives a $(k+l \alpha)$-dimensional Real manifold with boundary $M$. Therefore, $\varphi$ is well defined.

To show that the map is an isomorphism, consider the following commutative diagram:

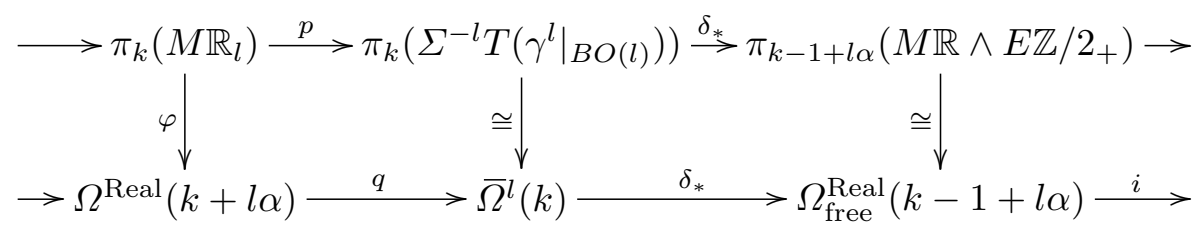


Here $q$ sends $M$ to $M^{\mathbb{Z} / 2}$ and $i$ sends the free Real cobordism class of a free manifold to its Real cobordism class. Thus, it suffices to show that the sequence of cobordism groups is exact, since then by the Five Lemma, the map $\varphi$ is an isomorphism. At $\bar{\Omega}^{l}(k)$, given a real manifold $M, q(M)=M^{\mathbb{Z} / 2}$. There is a tubular neighborhood $N^{\prime}$ of $M^{\mathbb{Z} / 2}$ in $M$ such that $N^{\prime} \cong E\left(\left.\nu\right|_{M^{\mathbb{Z} / 2}} ^{M}\right)$. Then

$$
\delta_{*} q(M)=\delta_{*}\left(M^{\mathbb{Z} / 2}\right)=S\left(\left.\nu\right|_{M^{\mathbb{Z} / 2}} ^{M}\right),
$$

which is cobordant to 0 via $M \backslash N^{\prime}$. Conversely, as we have seen above, given an $l$-trivial $N$ in $\bar{\Omega}^{l}(k)$ with $l$-dimensional bundle $\eta$, if its image is 0 in $\Omega_{\text {free }}^{\text {Real }}(k-1+l \alpha)$, then there is a free Real manifold $M^{\prime}$ with boundary $S(\eta)$. Thus, we can combine $D(\eta)$ with $M^{\prime}$ to get a Real manifold $M$ with $q: M \rightarrow N$. Hence, the sequence is exact at $\bar{\Omega}^{l}(k)$.

To show exactness at $\Omega_{\text {free }}^{\text {Real }}(k-1+l \alpha)$, suppose a free Real manifold $M$ is the image of some $l$-trivial $N$ with $l$-dimensional bundle $\eta$. Then $M$ is Real cobordant to $S(\eta)$, which in turn is Real cobordant to 0 via $D(\eta)$. Conversely, let $M$ be a free Real manifold of dimension $k-1+l \alpha$ with $i(M)=0$, so there is a Real manifold $P$ with boundary $M$. Then consider $P^{\mathbb{Z} / 2}$ in $\Omega^{X_{l}}(k)$ with

$$
\delta_{*}\left(M^{\mathbb{Z} / 2}\right)=S\left(\left.\nu\right|_{P} ^{P} / 2\right)
$$

in $\Omega_{\text {Real }}^{\text {free }}(k-1+l \alpha)$. Let $Q$ be a tubular neighborhood of $P^{\mathbb{Z} / 2}$ in $P, Q \cong$ $E\left(\left.\nu\right|_{P^{\mathbb{Z} / 2}} ^{P}\right)$. Then $M$ is cobordant to $S\left(\left.\nu\right|_{M^{\mathbb{Z} / 2}} ^{P}\right)$ via $P \backslash Q$, so $[M]=\delta_{*}\left[P^{\mathbb{Z} / 2}\right]$. Finally, the previous lemma gives exactness at $\Omega^{\text {Real }}(k+l \alpha)$.

\section{References}

[1] S. Araki, Orientations in $\tau$-cohomology theories, Japan J. Math. 5 (1979), 403-430.

[2] S. Araki and K. Iriye, Equivariant stable homotopy groups of spheres with involutions, I, Osaka J. Math. 19 (1982), 1-55.

[3] S. Araki and M. Murayama, $\tau$-cohomology theories, Japan J. Math. 4 (1978), 363-416.

[4] M. F. Atiyah, K-theory and Reality, Quart. J. Math. Oxford (2) 17 (1966), 367386.

[5] M. F. Atiyah, R. Bott and A. Shapiro, Clifford modules, Topology 3 (1964), suppl. 1, 3-38.

[6] M. F. Atiyah and G. B. Segal, Equivariant K-theory and completion, J. Differential Geom. 3 (1969) 1-18.

[7] P. E. Conner and E. E. Floyd, Differentiable Periodic Maps, Academic Press, New York, 1964.

[8] S. R. Costenoble and S. Waner, G-transversality revisited, Proc. Amer. Math. Soc. 116 (1992), 535-546.

[9] T. tom Dieck, Bordisms of $G$-manifolds and integrality theorems, Topology 9 (1970), 345-358. 
[10] M. Fujii, Cobordism theory with reality, Math. J. Okayama Univ. 18 (1976), 171188.

[11] - On the relation of real cobordism to KR-theory, ibid. 19 (1977), 147-158.

[12] - Bordism theory with reality and duality theorem of Poincaré type, ibid. 30 (1988), 151-160.

[13] I. Kriz, A Real analogue of the Adams-Novikov spectral sequence, in preparation.

[14] P. S. Landweber, Fixed point free conjugations on complex manifolds, Ann. of Math. (2) 86 (1967), 491-502.

[15] - Conjugations on complex manifolds and equivariant homotopy of $M U$, Bull. Amer. Math. Soc. 74 (1968), 271-274.

[16] L. G. Lewis, J. P. May and M. Steinberger, Equivariant Stable Homotopy Theory, with contributions by J. E. McClure, Lecture Notes in Math. 1213, Springer, Berlin, 1986.

[17] J. Milnor, Differentiable Topology, Princeton Univ. Press, 1958.

[18] J. Milnor and J. W. Stasheff, Characteristic Classes, Princeton Univ. Press and Univ. of Tokyo Press, 1974.

[19] R. E. Stong, Notes on Cobordism Theory, Princeton Univ. Press, 1968.

[20] A. G. Wasserman, Equivariant differential topology, Topology 8 (1969), 127-150.

Department of Mathematics

University of Chicago

5734 South University Avenue

Chicago, IL 60637, U.S.A

E-mail: pohu@math.uchicago.edu 